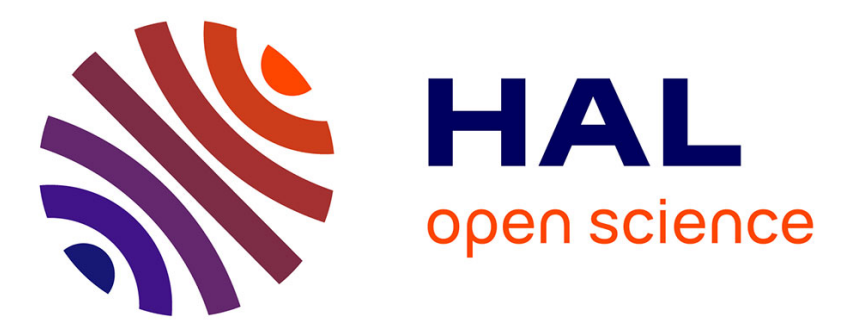

\title{
A Mobility Model Based on Improved Artificial Potential Fields for Swarms of UAVs
}

Ema Falomir, Serge Chaumette, Gilles Guerrini

\section{To cite this version:}

Ema Falomir, Serge Chaumette, Gilles Guerrini. A Mobility Model Based on Improved Artificial Potential Fields for Swarms of UAVs. IROS 2018 (International Conference on Intelligent Robots and Systems), IEEE, Oct 2018, Madrid, Spain. hal-01848857

\section{HAL Id: hal-01848857 \\ https://hal.science/hal-01848857}

Submitted on 25 Jul 2018

HAL is a multi-disciplinary open access archive for the deposit and dissemination of scientific research documents, whether they are published or not. The documents may come from teaching and research institutions in France or abroad, or from public or private research centers.
L'archive ouverte pluridisciplinaire HAL, est destinée au dépôt et à la diffusion de documents scientifiques de niveau recherche, publiés ou non, émanant des établissements d'enseignement et de recherche français ou étrangers, des laboratoires publics ou privés. 


\title{
A Mobility Model Based on Improved Artificial Potential Fields for Swarms of UAVs
}

\author{
Ema Falomir $^{1,2}$, Serge Chaumette ${ }^{1}$ and Gilles Guerrini ${ }^{2}$
}

\begin{abstract}
A combination of several autonomous UAVs can be used to perform collaborative tasks. Such a combination is referred to as a swarm of drones. The use of multiple platforms can extend the system global capacities thanks to the resulting variety of embedded sensors and to information sharing. In this case, path planning and thus obstacles avoidance is still a major task. To deal with this issue, mobility models have to be implemented. Our contribution presented in this paper is a mobility model for swarms of UAVs based on the Artificial Potential Fields (APF) principle. In our model, the involved UAVs collaborate by sharing data about the obstacles that they detected. By doing so, a UAV which is not close enough to an obstacle to detect it thanks to its own sensors will still have the proper data to take this obstacle into account in its path planning. To validate our mobility strategies with realistic constraints we simulate the performances of existing sensors and transmitters, and consider real-world environment.
\end{abstract}

\section{INTRODUCTION}

Plenty of studies have been made on Unmanned Aerial Vehicles (UAVs) in the last few years. Fixed wings and rotor wings (or multi-rotors) are the two main families of UAVs. Fixed wings are preferred for long flights but do not offer a lot of maneuverability. In this paper, we will consider multirotors. Indeed, in order to evolve in unknown environment, possibly containing obstacles, rotor wings are often preferred because they offer low speed maneuvering and hovering.

Unmanned Vehicles (UV) can be used to perform dull, dirty, dangerous and deep (4D) missions using electronics devices instead of humans, with low manufacturing cost, high adaptability or no risk of casualties. Furthermore, several UAVs can be used in a same Area of Interest (AoI) and fulfill together a single mission with better performances than with a single one (fulfill missions quicker or in larger areas for instance). Usage of a several vehicles instead of a single UAV can allow the system resilience; i.e. the lost of one or several vehicle(s) does not cause the mission failure. We talk of "multi-platform" or "swarm". The term "swarm" has no widely admitted definition, then we will use the one proposed in one of our previous papers[11]: a swarm refers to a set of UAVs with similar characteristics (speed, maneuvering etc.), able to establish peer-to-peer connections and can be considered as a single entity from an operator point-ofview. If this swarm is able to fulfill a mission without the intervention of an external (artificial or human) entity, then we qualify it of autonomous.

\footnotetext{
1 Univ. Bordeaux, LaBRI, UMR5800, F-33400 Talence, France \{efalomir, schaumette\}@u-bordeaux.fr

${ }^{2}$ Thales DMS France, F-33700 Mérignac, France $\{$ ema.falomir, gilles.guerrini\}@fr.thalesgroup.com
}

A main task of UAVs collaboration is path planning, including collision avoidance. The Artificial Potential Fields (APF) principle is a famous path planning method that allows smooth trajectory generation and do not require a search of a global path. Furthermore, it is highly efficient and has a computational cost low enough to be performed on embedded systems [19]. Our model is based on this principle, and details of APF will thus be given in section III along with alternative methods.

In this paper, a mobility model for autonomous swarms of UAVs based on APF is presented. It allows collaboration between the platforms and collision avoidance. Problems of unreachable targets and local minima have been solved and an innovative repulsive potential field allows anticipation of obstacle avoidance. The method is validated with several simulations using the network simulator OMNeT++ [4].

This paper is composed of the following sections: section II introduces possible applications of UAVs swarms and their embedded sensors. Section III presents a state of the Art of mobility strategies for UAVs especially using APF. Our model is described in section IV as well as the method hypothesis and pseudo-code. Finally, results of several simulations are analyzed in section $\mathrm{V}$, before conclusions and perspectives in section VI.

\section{Applications of UAVs SWARMS AND EMBEDDED SENSORS}

In this paper we focus on UAVs of the multi-rotors type, with a width of approximately 50 centimeters. This section presents some applications of swarms of UAVs, and the sensors that they can carry.

\section{A. Applications of Multi-UAV Systems}

Swarms of UAVs have many civil applications. For instance, they are used to help firemen in the case of large forest fires [20], to spray pesticides on crops [10], or to survey points of interest [17] or AoIs [15].

In frame of military activities, some studies [5] have been done about UAVs swarms in contexts of collaborative mission planning for Intelligence, Surveillance and Reconnaissance (ISR) missions. Path planning for multi-platform have also been studied [21], including embedded sensors capacities, in environments containing obstacles.

\section{B. Radio Module}

To fulfill collaborative tasks, the involved UAVs should share information, for example concerning their environment. To do that autonomously, they have to carry a radio module. 
XBee platforms are communication modules that suit well the applications of UAVs because they are small, have a light weight, require little energy and are easily customizable and programmable [1]. They are often used on multi-rotors, see for example [17], [26].

In our application, we simulate the characteristics of a XBee-PRO module [3]. In our study the two key characteristics are the data rate $(250 \mathrm{Kbps})$ and the communication range in urban environment (90 meters).

\section{Obstacle Detection}

There are three major technologies to deal with obstacle detection for the kind of small scale drones that we are addressing: SONAR (SOund Navigation And Ranging), RADAR (RAdio Detection And Ranging) and LI DAR (LIght Detection And Ranging). In the following, we discuss the characteristics of each of them in the context of our scenario, and select the most adapted.

SONAR technology relies on the uptake of the reflection of a sound wave by a distant object in a given angular area. SONARs are cheap, light and low-power. However this technology is not appropriate for long range aerial detection with a maximum range of 10 meters, which is far from enough for our scenario.

RADARs use radio waves instead of sound waves, and so allow long-distance obstacles detection. Nevertheless, only small ones can be carried by multi-rotors and they have a level of resolution too low for our application.

The LIDAR technology relies on the use of a laser beam focused to a tight spot, thus providing a high level of precision. Compact portable LIDARs can be used to detect objects located at several tens of meters. As LIDARs capture one measure per spot, they generates a lot of measurements. They thus need more computational resources to deal with.

We have decided to use a LIDAR because of the precision and of the distance to potential obstacles in the scenario that we address. We have selected the LIDAR-Lite v3 by Garmin which has a range of $40 \mathrm{~m}$, a resolution of $1 \mathrm{~cm}$ and a correct accuracy (when considering obstacle detection). Its update rate is high (up to $500 \mathrm{~Hz}$ ), so we can mount it on a servomotor in order to get information on wide angles.

\section{Network Simulator: OMNeT++}

In order to simulate the characteristics of the sensor and transmitter presented above, we chose the network simulator OMNeT++ [4]. It allows numerous degrees of realism in the simulations, from ideal environment and performances of each element, to the modeling of energy consumption, antennas parameters or signal propagation. The main communication parameters that we chose are given in appendix.

\section{RELATED WORK}

Many path planning methods for UVs exist. In this section we will first focus on trajectory planning for UAVs, and then on planning methods based on APF and finally on methods designed for multi-platform systems.

\section{A. Path Planning for Autonomous UAVs}

A task of major importance for autonomous UVs is path planning. This task is of increasing complexity if the environment in which the drones operate are not known prior to the mission (and contain obstacles) and thus need to be calculated on board, in real-time. Some methods have been extensively studied to solve this problem: APF methods introduced by Khatib [16], that we detail in the following section; virtual forces [11],[13]; Genetic Algorithms [14]; chaotic processes [23]; fuzzy logic [9]; Particule Swarm Optimization (PSO) or Reinforcement Learning [8].

\section{B. Path planning for UVs based on APFs}

The APF method introduced by Khatib [16] has numerous successors today. Indeed, this methods has some drawbacks as "Goal Non Reachable with Obstacle Nearby" (GNRON), local minima, oscillations close to obstacles (jitter problem) or the impossibility to go between two close obstacles [25].

Sun et al. [25] propose a collision avoidance method based on APFs for cooperative UAVs, with a local adjustment to overcome the jitter problem, and potential fields definition removing the other problems (GNRON, local minima and impossibility to go between two close obstacles). The collaboration process is not explained in this paper.

Additionally to the issues cited above, we can quote the consideration of obstacles as points, which can be used only for the small ones. Mac et al. [19] overcame this problem by considering large obstacles as a sum of punctual ones. In this paper, they also proposed a solution to the GNRON problem. This approach seems to be efficient for a single UAV, but an important part of our work consists in using collaboration between drones to improve the the model performances. The model defined by Mac et al. is not designed for swarming and consequently we cannot use it as is.

The local minima problem can be solved by creating a temporary and virtual waypoint, as presented by Liu and Zhao [18]. In order to reduce the number of local minima, they consider close obstacles as one single larger obstacle. There is no simulation showing what happens if the goal is inside the envelope of several close obstacles. Furthermore, some of the other problems listed above are not mentioned.

Thanks to the several ways to solve the traditional APF method problems, it is possible to use this kind of algorithms for trajectory planning adapted to single and multi-platform, as shown by Galvez et al. [12]. In their paper, they use APF to maintain several quadrotors in formation on their way (possibly containing obstacles) towards a target point. Note that our objectives do not include formation control, then their model is not adapted to our scenario.

Finally, in all the improved APF that we found in the literature, the repulsive field linked to obstacles is symmetrical. We believe that it should depend on the UV and target point locations. Indeed, if a UV is close to its target, it is useless to consider a repulsive potential field due to an obstacle located at the back of the vehicle. Similarly, if an obstacle is not on the path towards the target point of a UV, it should not have 
any influence on the vehicle path planning. In our model, we reduce the repulsive field to the safety distance if the obstacle is not on the UAV path; if it is, then it has a great influence, as detailed in section IV-F.1.

\section{Path Planning for Multi-Platform Systems}

Some path planning methods are particularly adapted for multi-platform systems.

For example, we can quote Ant Colony Optimization methods (introduced in [7], widely studied since then [6]) which are especially efficient for area coverage missions but this is not our main target scenario.

Models based on Boids are also widely studied. They allow stabilization of compact formations, even in environments containing obstacles [24]. Rosalie et al. [22] recently proposed a model combining Boids principle, Ant Colony Optimization and chaotic dynamics to fulfill a mission of area coverage while maximizing connectivity within the swarm.

Finally, we can quote Virtual Forces methods, that make it possible to maintain formations in constrained environments or to fulfill coverage missions [11]. Virtual Forces are also adapted for systems that rely on leaders and followers [13]. Still, methods based on virtual forces have problems similar to those of APF-based methods.

\section{Our Mobility Model}

\section{A. Hypothesis}

At the beginning of the mission (or simulation), all UAVs are inside the AoI. They have to reach a common target point, which is also inside the AoI. The UAVs always instantly know with sufficient precision their own location, the location of the target and the limits of the AoI. The UAVs evolve in a discrete environment. At each iteration, they can either move to the center of one of the 8 adjacent square-cell, or stay at the same location. No mobile obstacle is considered in this study. Each UAV carries a sensor allowing obstacles detection (fix obstacles or other UAVs) which are located in a given range (see section II-C) at $360^{\circ}$. In our model, the cells width must be greater than this sensor range. The UAVs can communicate if the distance between them is smaller than the radio range (see section $\mathrm{II}-\mathrm{B}$ ).

\section{B. Notations}

The environment in which the UAVs move is meshed. All the cells are squares of equal size. Hence, we consider a matrix of $l_{\text {max }}$ lines and $c_{\max }$ columns, both finite numbers. We note $c_{i, j}$ the cell in line $i$ and column $j$ where, $(i, j) \in$ $\llbracket 1, l_{\max } \rrbracket \times \llbracket 1, c_{\max } \rrbracket$. We note $t_{i, j}$ the cell in line $t_{i}$ and column $t_{j}$ containing the target. Likewise, $\operatorname{uav}_{i, j}$ represents the cell containing a UAV in line $\mathrm{uav}_{i}$ and column $\mathrm{uav}_{j}$.

Three matrices are considered for this model, each of size $l_{\max } \times c_{\max }$. The first one represents the potential field and is noted pot. The second stores the locations of known fix obstacles; it is noted obs. The third is used to anticipate the fix obstacle avoidance (see below) and for the UAV avoidance, and is noted avoid.

\section{Principle}

At each iteration, each UAV moves to the adjacent cell with the smallest potential.

If an obstacle is (even partially) in a cell, the potential of this cell is increased by a fixed positive value called obsInc.

\section{Matrices Meanings and Initializations}

The matrix obs is the only one exchanged by the UAVs. Each cell of this matrix has a value representing if:

- there is no information about the cell;

- it does not contains an obstacle and is safe;

- it does not contains an obstacle but is not safe or has been revisited;

- it contains an obstacle;

- it is on a path already used by a UAV to reach the target.

The matrix noted pot contains the APF related to the target point, the APF related to the obstacles which have been detected and the potential related to cells which have already been visited.

The matrix noted avoid stocks the potential related to the anticipation of fix obstacles avoidance and the temporary potential linked to neighbors. It is the only one that is reinitialized at each iteration.

The potential related to the target is initialized with the distance between the cell and the target (in cell $t_{i, j}$ ), using the infinity norm, as given in the following equation:

$$
\operatorname{pot}_{i, j}=\max \left(\left|t_{i}-i\right|,\left|t_{j}-j\right|\right) .
$$

At the beginning of the mission, the UAVs have no information on the AoI (except its limits). Then, there is no potential linked to obstacles, all the cells of the obs matrix represent "no information" and the avoid matrix is null.

\section{E. Collision Avoidance}

1) Collision Avoidance UAV-fix obstacle: We consider a collision if a UAV is in a cell containing an obstacle. Hence, when an obstacle is detected, the potential of its cell is increased by a fixed value noted obsInc and all the adjacent cells are increased by obsInc/2.

2) Collision Avoidance $U A V-U A V$ : At each iteration, the UAVs detect their neighbors in their sensor range. UAVs consider each other as a temporary obstacle, and apply the same increases as with fix obstacles: increase by obsInc on the neighbor cell and obsInc/2 on the adjacent cells. These potential increases are recorded in the avoid matrix, which is re-initialized at each iteration, because the neighbors location can change at each iteration.

\section{F. Algorithm}

1) Obstacle Avoidance Anticipation: In the case of the traditional APF, the influence of an obstacle is symmetrical. In our mobility strategy, an obstacle has an influence on a UAV while it is on its way towards the target point. In other words, if a UAV has information about an obstacle located between itself and its target point, it will increase the potential of all the cells between itself and the obstacle. Fig. 1 shows the variations of potential in this case. 


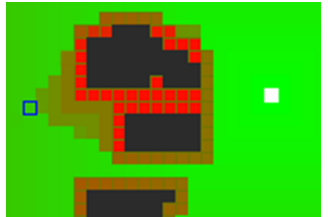

Fig. 1. Illustration of the potential field in the case of obstacle avoidance anticipation. UAV is represented by a dark empty square, the obstacle is represented in dark blue. The cells colored in green are not submitted to any increase. The cells submitted to a potential increase are represented with gradation from brown (smallest) to red (highest).

Data: $\operatorname{uav}_{i, j}, t_{i, j}, o_{i, j}{ }^{*}$

Calculate min and max line and column of the whole

obstacle: $o_{i M i n}, o_{i M a x}, o_{j M i n}, o_{j M a x}$;

Calculate min and max oriented angle

obstacle-UAV-target: $a_{\mathrm{Min}}, a_{\mathrm{Max}}$;

for $i \leftarrow \min \left(\operatorname{uav}_{i}, o_{i M i n}\right)$ to $\max \left(\operatorname{uav}_{i}, o_{i M a x}\right)$ do

for $j \leftarrow \min \left(\operatorname{uav}_{j}, o_{j M i n}\right)$ to $\max \left(\operatorname{uav}_{j}, o_{j M a x}\right)$

do

if $a_{\mathrm{Min}}<\operatorname{angle}\left(c_{i, j}, \mathrm{uav}_{\mathrm{i}, \mathrm{j}}, g_{i, j}\right)<a_{\mathrm{Max}}$ and $\operatorname{dist}\left(c_{i, j}, o_{i, j}\right)<\operatorname{dist}\left(\operatorname{uav}_{\mathrm{i}, \mathrm{j}}, o_{i, j}\right)$ then $\operatorname{avoid}_{i, j}=\operatorname{ceil}\left(\frac{\mathrm{obsInc}}{\operatorname{dist}\left(\left(c_{i, j}, o_{i, j}\right)+1\right.}\right)$; end

end

end

Algorithm 1. Obstacle Avoidance Anticipation (OAA)

${ }^{*} o_{i, j}$ is the considered cell containing an obstacle on the way toward the target

Data: $\operatorname{uav}_{i, j}$, pot matrix;

avoid $\leftarrow 0_{l_{\max }, c_{\max }}$;

if $\operatorname{uav}_{i, j}$ has already been visited then

$\operatorname{pot}_{i, j} \leftarrow \operatorname{pot}_{i, j}+1^{*}$;

else

| Add uav $i, j$ to visited cells;

end

Detect fix obstacles;

foreach obstacle stored in avoid do

| OAA $\left(\mathrm{uav}_{\mathrm{i}, \mathrm{j}}, g_{i, j}, o_{i, j}\right)$;

end

Detect other UAVs and increase potential around them;

Calculate the minimum potential of the adjacent cells**;

Store the cell with minimum potential in $m_{\text {minPot }}$;

if $m_{\operatorname{minPot}}=\mathrm{uav}_{i, j}$ then

| $\operatorname{pot}_{i, j}=$ mean of adjacent cells;

end

Next cell $\leftarrow m_{\operatorname{minPot}}$;

ALGORITHM 2. Move decision by each UAV.

* The increase is of 1 because it is the difference between the potential of two adjacent cells when there is no influence of obstacles. Then, it is enough to favor the exploration of other cells, and is small enough not to interfere with obstacles (anticipation) avoidance.

** If several of these cells have the smallest potential, the one closest to the target is recorded.
This anticipation of obstacle avoidance is particularly efficient if the UAVs can detect obstacles at a long distance, or if they get information from other sources (other UAVs in our case). UAVs have information on obstacle presence cell by cell. Then, if one cell containing an obstacle is on their way towards the target, they verify the presence in all the cells at a distance 2 (calculated with the infinity norm) to deduce the whole obstacle location. We consider a safety distance around obstacle of one cell, then two obstacles separated by 2 cells are considered as a single one, as shows Fig. 1. Algorithm 1 is the pseudo-code describing this process.

2) Mobility Strategy: The motion decision of a UAV at each iteration depends on the location of its target point, of its close neighbors and on the discovered obstacles, as detailed in algorithm 2.

When a UAV reaches the target point, it sets the cells of the avoid matrix corresponding the ones crossed to "on a way already used to reach the target". When the other UAVs receive this information, they decrease the potential of theses cells by a fix value. Then, if a UAV is on a cell on a way to the target, it will follow this path which ensures the arrival.

\section{Simulations}

\section{A. Simulated Environment}

One application of our model is the identification of an intruder in restricted areas. Then, we used the open source French land register [2] to reproduce the buildings of the Blayais nuclear power station (France). We focused on a part of this area of $500 \mathrm{~m}$ large and $500 \mathrm{~m}$ long, as shows Fig. 2.

\section{B. Parameters}

As the considered UAVs are $0.5 \mathrm{~m}$ large, we state a $1.5 \mathrm{~m}$ safety distance. Then, the minimal cell size of the mesh is $2 \mathrm{~m}$. Considering the $500 \mathrm{~m}$ times $500 \mathrm{~m}$ area, the obstacles matrix has 250 lines and 250 columns. Each cell of this matrix contains one of the 5 different states described in section IV-D, represented by integers. We used integers coded on 8 bits, then the data in the matrix is coded on 500kbit. The

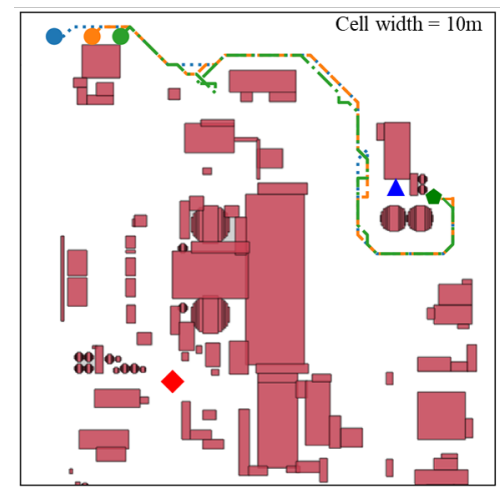

Fig. 2. Environment of simulations. Obstacles are represented in red. Trajectories of the 3 UAVs composing the swarm are plotted from their initial location (circles) toward their target point called T2 (represented by a green pentagon). The target point $\mathrm{T} 1$ is represented by a red square and $\mathrm{T} 3$ by a blue triangle. 


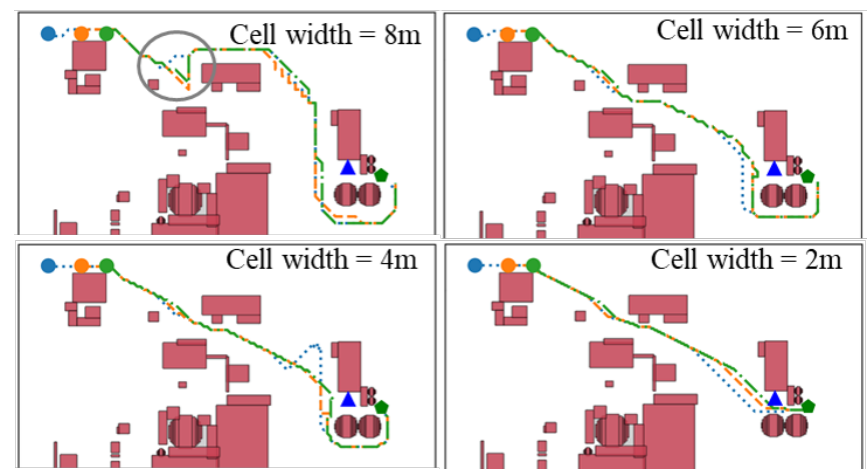

Fig. 3. The trajectories of the 3 UAVs composing the swarm from their initial location toward their target point $\mathrm{T} 2$.

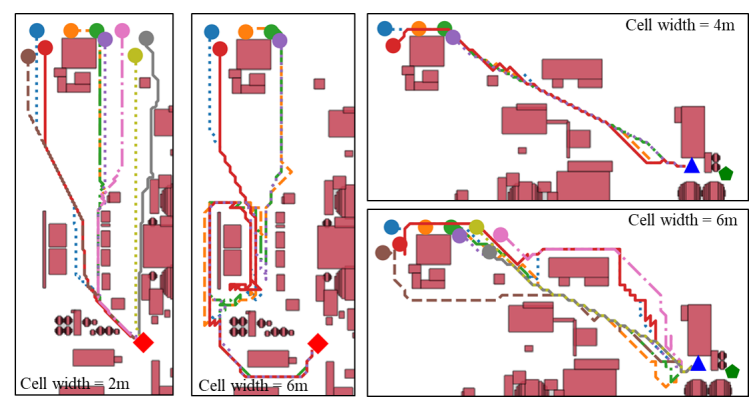

Fig. 4. Example of UAVs trajectories with targets T1 and T3, with various cell width.

XBee data rate is up to $250 \mathrm{kbit} / \mathrm{s}$ and the communication is half-duplex. Thus a single UAV can broadcast its updated matrix every two seconds. Even though collaboration allows the UAVs to reach the target quicker, the low communication rate is not a safety issue as UAVs do not require collaboration to evolve safely towards the target. The simulated sensor for obstacle detection is a LIDAR-Lite (see section II-C), with a detection range up to $40 \mathrm{~m}$. We suppose that it is mounted on a platform allowing detection all around the UAV. So as to test the model in different conditions, we performed the simulations using multiple cell widths $(2,4,6,8$ and $10 \mathrm{~m})$, several swarms sizes (3, 5, 7 and $9 \mathrm{UAVs})$, and with three different targets.

\section{Collision Avoidance}

For all the simulations performed, the distance between a UAV and another object (building or UAV) was greater than half of the cell width, so there were no any collision.

\section{UAVs Trajectories}

Fig. 2 and 3 represent the trajectories of the 3 UAVs composing a swarm moving toward the target point $\mathrm{T} 2$ using different cell widths. The part of the trajectory circled in the case of a $8 \mathrm{~m}$-large cell illustrates well the collaboration between the UAVs: a first one detects an obstacle, avoids it and transmit the information to the others that anticipate the best path toward the goal.

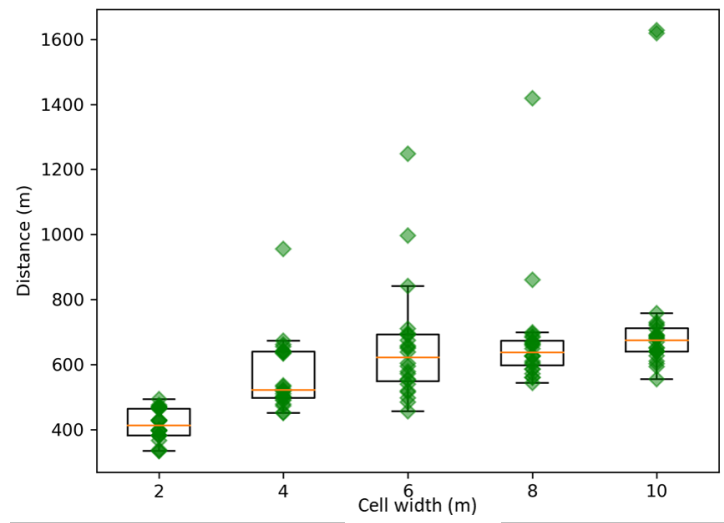

Fig. 5. Superposition for a given cell width (2, 4, 6, 8 and 10) of the distances traveled towards the target $\mathrm{T} 2$ by each individual UAV in swarms of $3,5,7$ and 9 vehicles,.

It has to be noted that our mobility strategy allows the UAVs to reach all these targets, including surrounded by obstacles (case of target T3). In other words, our model solves the "Goal Non Reachable with Obstacle Nearby" (GNRON) problem. Furthermore, our model also allows the UAV to go between two close obstacles (Fig 3 and 4).

\section{E. Influence of the Cell Width}

Cell width is an important parameter in our model. First, we consider that a cell is either empty or filled with an obstacle since a building has at least one square meter (precision of the environment model) within it. The larger the cells, the larger the forbidden areas. This leads in one hand to longer path to avoid these areas (see Fig. 3 and 5) and on the other hand to non reachable goals as in the case of T1 and T3 for cells larger than 6 meters. Fig. 5 represents the travel distance of UAVs from their launch point to the target T2 with swarms from 3 to 9 UAVs. It shows that the smaller the cell, the shortest the path to the goal.

Furthermore, fewer iterations are required to travel the same distance with large cells than with small ones and so the process is less calculation intensive. Also, the larger the cell, the smaller the matrices. The messages exchanged between the UAVs are thus smaller with large cells.

Finally, the UAVs travel from a cell to an adjacent one at each iteration. Then, the larger the cell, the longer the distance at each iteration, and so the quicker at the target.

\section{CONCLUSiOn AND Future Work}

This paper presents a new mobility strategy for autonomous swarms of collaborative UAVs. Our method uses the principle of APF but differs from the traditional one: in our solution, the influence of obstacles depends on the locations of the UAVs and the location of their target. This allows anticipation of obstacles avoidance and solve the "Goal Non Reachable with Obstacle Nearby" problem. We validated the efficiency of our mobility strategy with simulations under OMNeT++, in a realistic environment and with the simulation of existing sensors performances. 
In future work for the optimization of our method we will consider moving obstacles and simulate the 3 dimensions. Furthermore, we plan to combine these potential fields with a global path planning algorithm (such as $\mathrm{A}^{*}$ ), in order to shorten the UAVs trajectories.

\section{APPENDIX}

Main Communication Parameters used is OMNeT++:

- Wireless Local Area Network (WLAN)

- type name: "IdealWirelessNic"

- communication range: $90 \mathrm{~m}$

- full duplex: false

- Routing

- destination address: 10.0.255.255

- forwarding: false

- optimize routes: false

- UDP application

- type name: own model based on "UDPBasicApp"

- send interval: $500 \mathrm{~ms}$

- message length: 62500B (see section V-B)

- bitrate: 250kbits/s

- Radio

- radio type: "APSKScalarRadio"

- radio medium type: "APSKScalarRadioMedium"

- carrier frequency: $2.4 \mathrm{Ghz}$

- bandwith: $2 \mathrm{MHz}$

- background noise power: $-90 \mathrm{dBm}$

- transmitter power: $63 \mathrm{~mW}$

- receiver sensitivity: $-102 \mathrm{dBm}$

- receiver snir threshold: $4 \mathrm{~dB}$

- receiver ignore interference: false

- transmitter header bit length: 192b

- Environment

- ground type: "FlatGround"

- ground elevation: $0 \mathrm{~m}$

- path loss type: "TwoRayGroundReflection"

\section{REFERENCES}

[1] Digi XBee Ecosystem - Everything you need to explore and create wireless connectivity.

[2] Géoportail.

[3] XBee $₫$ \& XBee-PRO $®$ ZB ZigBee $₫$ Embedded RF Module Family for OEMs, 2011.

[4] Varga András and Hornig Rudolf. An overview of the OMNeT++ simulation environment. In Proceedings of the 1st international conference on Simulation tools and techniques for communications, networks and systems \& workshops, pages 1-10, Marseille, France, March 2008. ICST (Institute for Computer Sciences, Social-Informatics and Telecommunications Engineering).

[5] Jovan Boskovic, Nathan Knoebel, Nima Moshtagh, Jayesh Amin, and Gregory Larson. Collaborative Mission Planning \& Autonomous Control Technology (CoMPACT) System Employing Swarms of UAVs. American Institute of Aeronautics and Astronautics, August 2009.

[6] Ugur Cekmez, Mustafa Ozsiginan, and Ozgur Koray Sahingoz. Multi colony ant optimization for UAV path planning with obstacle avoidance. In 2016 International Conference on Unmanned Aircraft Systems (ICUAS), pages 47-52. IEEE, June 2016.

[7] Alberto Colorni, Marco Dorigo, and Vittorio Maniezzo. Distributed Optimization by Ant Colonies. In European Conference on Artificial Life, pages 134-142, Paris, France, 1992. ELSEVIER.

[8] Ezequiel Di Mario, Zeynab Talebpour, and Alcherio Martinoli. A comparison of PSO and reinforcement learning for multi-robot obstacle avoidance. In Evolutionary Computation (CEC), 2013 IEEE Congress on, pages 149-156. Ieee, 2013.
[9] Biqiang Du and Shizhao Liu. A common obstacle avoidance module based on fuzzy algorithm for Unmanned Aerial Vehicle - IEEE Conference Publication. pages 245-248. IEEE, June 2016.

[10] Bruno S. Faiçal, Gustavo Pessin, Geraldo P. R. Filho, André C. P. L. F. Carvalho, Gustavo Furquim, and Jó Ueyama. Fine-Tuning of UAV Control Rules for Spraying Pesticides on Crop Fields. In 2014 IEEE 26th International Conference on Tools with Artificial Intelligence, pages 527-533, November 2014.

[11] Ema Falomir, Serge Chaumette, and Gilles Guerrini. Mobility Strategies based on Virtual Forces for Swarms of Autonomous UAVs in Constrained Environments. volume 1, pages 221-229, July 2017.

[12] Regan L. Galvez, Gerard Ely U. Faelden, Jose Martin Z. Maningo, Reiichiro Christian S. Nakano, Elmer P. Dadios, Argel A. Bandala, Ryan Rhay P. Vicerra, and Arvin H. Fernando Fernando. Obstacle avoidance algorithm for swarm of quadrotor unmanned aerial vehicle using artificial potential fields. In TENCON 2017 - 2017 IEEE Region 10 Conference, pages 2307-2312, November 2017.

[13] Johannes Gobel and Anthony E. Krzesinski. A model of autonomous motion in ad hoc networks to maximise area coverage. In Telecommunication Networks and Applications Conference, 2008. ATNAC 2008. Australasian, pages 258-263. IEEE, 2008.

[14] Nikolos Ioannis K., Valavanis Kimon P., and Tsourveloudis Nikos C. Evolutionary algorithm based offline/online path planner for UAV navigation. IEEE Transactions on Systems, Man, and Cybernetics, Part B (Cybernetics), 33:898-912, December 2003.

[15] Aldo Jaimes, Srinath Kota, and J. Gomez. An approach to surveillance an area using swarm of fixed wing and quad-rotor unmanned aerial vehicles UAV(s). In 2008 IEEE International Conference on System of Systems Engineering, pages 1-6, June 2008.

[16] Oussama Khatib. Real-time obstacle avoidance for manipulators and mobile robots. The international journal of robotics research, 5(1):9098, 1986.

[17] Rémi Laplace. Applications et services DTN pour flotte collaborative de drones. PhD Thesis, Université Sciences et Technologies-Bordeaux I, 2012.

[18] Yuecheng Liu and Yongiia Zhao. A virtual-waypoint based artificial potential field method for UAV path planning. In Guidance, Navigation and Control Conference (CGNCC), 2016 IEEE Chinese, pages 949953. IEEE, 2016.

[19] Thi Thoa Mac, Cosmin Copot, Andres Hernandez, and Robin De Keyser. Improved potential field method for unknown obstacle avoidance using UAV in indoor environment. In Applied Machine Intelligence and Informatics (SAMI), 2016 IEEE 14th International Symposium on, pages 345-350. IEEE, 2016.

[20] Ivan Maza, Fernando Caballero, Jesus Capitan, Jose Ramiro Martinezde Dios, and Anibal Ollero. Firemen monitoring with multiple UAVs for search and rescue missions. In 2010 IEEE Safety Security and Rescue Robotics, pages 1-6. IEEE, July 2010.

[21] Joshua Redding, Jovan Boskovic, Raman Mehra, and Chunlei Rui. Heterogeneous Cooperative Control of Multiple UAVs with Collaborative Assignment and Reactive Motion Planning. American Institute of Aeronautics and Astronautics, August 2008.

[22] Martin Rosalie, Matthias R. Brust, Gregoire Danoy, Serge Chaumette, and Pascal Bouvry. Coverage Optimization with Connectivity Preservation for UAV Swarms Applying Chaotic Dynamics. pages 113-118. IEEE, July 2017.

[23] Martin Rosalie, Grégoire Danoy, Serge Chaumette, and Pascal Bouvry. From random process to chaotic behavior in swarms of UAVs. DIVANet '16 Proceedings of the 6th ACM Symposium on Development and Analysis of Intelligent Vehicular Networks and Applications, pages 9-15, Malta, November 2016.

[24] Martin Saska, Jan Vakula, and Libor Přeućil. Swarms of micro aerial vehicles stabilized under a visual relative localization. In Robotics and Automation (ICRA), 2014 IEEE International Conference on, pages 3570-3575. IEEE, 2014.

[25] Jiayi Sun, Jun Tang, and Songyang Lao. Collision Avoidance for Cooperative UAVs With Optimized Artificial Potential Field Algorithm. IEEE Access, 5:18382-18390, 2017.

[26] C. C. Zhih, S. K. V. Ragavan, and M. Shanmugavel. Development of a simple, low-cost autopilot system for multi-rotor UAVs. In 2015 IEEE Recent Advances in Intelligent Computational Systems (RAICS), pages 285-289, December 2015. 